\title{
Dynamical Analysis of Fractional-Order Hastings-Powell Food Chain Model with Alternative Food
}

\author{
Moh. Nurul Huda ${ }^{1}$, Trisilowati $^{2}$, Agus Suryanto \\ ${ }^{1}$ Master Program of Mathematics, University of Brawijaya, Malang, Indonesia \\ ${ }^{2}$ Department of Mathematics, Faculty of Mathematics and Natural Sciences, University of Brawijaya, Malang, Indonesia
}

Abstract

In this paper, a fractional-order Hastings-Powell food chain model is discussed. It is assumed that the top-predator population is supported by alternative food. Existence and local stability of equilibrium points of fractional-order system are investigated. Numerical simulations are conducted to illustrate analysis results. The analysis results show that alternative food can give a positive impact for top-predator population.

Keywords: Alternative food, Fractional-order, Grunwald-Letnikov approximation, Hasting-Powell model, Stability.

\section{INTRODUCTION}

Nowadays, fractional calculus becomes the main focus for the researchers. Many problems of science and engineering can be modeled by using fractional derivatives. The process of developing a differential system of integer order into fractional-order becomes popular in dynamic systems [1]. Basically, a biological mathematical model in predicting the future, not only depends on the current but also the memory or the previous condition. In fractional derivatives, at some certain conditions contain information of previous condition, therefore fractional derivatives can be used to explain more realistic natural phenomena.

Interactions between populations can be described in a food chain model. One of the interactions in the food chain is predation process. Mathematical model used to describe interactions between predator and prey is called the predator-prey model. Furthermore, many interesting fenomena in ecology can be described by mathematical model through predator-prey models such as harvesting in predator population [1], supplying alternative food in a predator population [2], refuging prey population [3], spreading disease in ecosystem [4], and the effect of the present an omnivore [5]. In predator prey model [2], it is assumed that prey populations do not always exist, they also experience migration to find new habitats due to climate change factors and low food reserves.

\footnotetext{
* Correspondence author:

Trisilowati

Email : trisilowati@ub.ac.id

Address : Faculty of Mathematics and Natural Sciences, University of Brawijaya, Jl. Veteran Malang, 65145.
}

Therefore, predators need additional food or alternative prey to survive.

In this paper, a food chain model of threespecies fractional-order with alternative food is introduced. Examples of three-species ecosystems in this model: vegetation-hare-lynx, mouse-snake-owl and worm-robin-falcon. Moreover, predator-prey food chains have been studied in structured populations in $[5,6]$. In this paper, Model is a modification of model [7]. Then the conditions of existence and stability of the equilibrium points of the fractional-order are examined in the result and discussion. Numerical simulations are illustrated by the GrunwaldLetnikov approximation [8].

\section{MATERIALS AND METHODS \\ Model Formulation}

In this research, the predatory-prey model [7] is the main object of the study. The model construction is done by modifying the model of Sahoo and Poria [2] by changing the integer order into the fractional-order.

\section{Determination of the Equilibrium Point}

In dynamical analysis, the first step is to determine the equilibrium points. The equilibrium point is obtained when the population rate of the system is unchanged or zero. From this condition, the existence properties of each equilibrium points is also obtained.

\section{Stability of the Equilibrium Point}

In this paper, the local stability of equilibrium points is analyzed. The discussion of local stability is begun by linearizing the model by using Taylor series. The linearization around its equilibrium 
point is done to change the nonlinear model into linear form. Approximation of linear system using Taylors series will be in the form of Jacobian matrix. From the Jacobian matrix, it is determined the roots of the characteristic equation or eigenvalue. The determination of local stability can be obtained from the absolute of its eigen value argument.

\section{Numerical Simulation}

The behavior of the system [3] is described by numerical simulation. The numerical simulation approach uses the Grunwald-Letnikov scheme. The numerical simulations are conducted by using MATLAB software. An important step in this stage is to determine parameters that match the condition of existence and the stability of the equilibrium points. The behavior of local stability is visualized by graphic based on kinds of parameter values pointed. The last step at this stage is do the interpretation results of numerical simulation.

\section{RESULT AND DISCUSSION}

Model Formulation

In this model, definition of Caputo fractional derivative is used. In [9] Caputo's definition of fractional derivatives can be written as follows

$$
\begin{aligned}
D_{t}^{\alpha} f(t) & =I_{t}^{n-\alpha} D_{t}^{n} f(t), \quad n=1,2, \ldots \\
& =\frac{1}{\Gamma(n-\alpha)} \int_{a}^{t}(t-s)^{n-1-\alpha} f^{(n)}(s) d s,
\end{aligned}
$$

with $n-1<\alpha<n$ and $\Gamma$ is a Gamma function and $D_{t}=\frac{d}{d t}$.

Hastings and Powell [10] has discussed the food chain model of three species. The three species are prey population $(X)$, intermediatepredator $(Y)$ and top-predator $(Z)$. The prey population is hunted by the intermediatepredator population and the top-predator hunts intermediate-predators. Both predation processes use the Holling Type II response function. $R_{0}$ and $K_{0}$ express the rate of growth and carrying capacity of the prey population. $C_{1}$ and $C_{2}$ are the interaction rates between prey populations, intermediate-predators with predators, toppredators. $B_{1}$ and $B_{2}$ are the rate of environmental protection to the prey and the intermediate-predator populations. $A_{1}$ and $A_{2}$ are the maximal predation rate of intermediatepredator and top-predator populations. $D_{1}$ and $D_{2}$ are the natural death rates of intermediatepredator and top-predator populations respectively. Mataouk et al. [10] modified the Hastings-Powell model [8] by changing the integer order into the fractional-order as follows:

$$
\begin{gathered}
D_{t}^{\alpha} X=R_{0} X\left(1-\frac{X}{K_{0}}\right)-\frac{C_{1} A_{1} X Y}{B_{1}+X}, \\
D_{t}^{\alpha} Y=\frac{A_{1} X Y}{B_{1}+X}-\frac{A_{2} Y Z}{B_{2}+Y}-D_{1} Y, \\
D_{t}^{\alpha} Z=\frac{C_{2} A_{2} Y Z}{B_{2}+Y}-D_{2} Z,
\end{gathered}
$$

with $0<\alpha<1$.

Model (1) explains that top-predator food sources only depend on intermediate-predators. However, alternative prey (supplementary feeding) for top-predators can reduce predation rates in intermediate-predators [2], then to give this effect, model (1) can be modified to

$$
\begin{gathered}
D_{t}^{\alpha} X=R_{0} X\left(1-\frac{X}{K_{0}}\right)-\frac{C_{1} A_{1} X Y}{B_{1}+X} \\
D_{t}^{\alpha} Y=\frac{A_{1} X Y}{B_{1}+X}-\frac{A A_{2} Y Z}{B_{2}+Y}-D_{1} Y \\
D_{t}^{\alpha} Z=C_{2} A_{2} Z\left(\frac{A Y}{B_{2}+Y}+(1-A)\right)-D_{2} Z
\end{gathered}
$$

where $A$ is a time independent constant to get the alternative resource $(0<A<1)$. To make easier the model analysis, variables and some parameter are selected to be

$$
\begin{aligned}
& x=\frac{X}{K_{0}}, y=\frac{C_{1} Y}{K_{0}}, z=\frac{C_{1} Z}{C_{2} K_{0}}, \quad t=R_{0} T, a_{1}= \\
& \frac{A_{1} K_{0}}{R_{0} B_{1}}, \quad a_{2}=\frac{A_{2} K_{0} C_{2}}{B_{2} R_{0} C_{1}}, b_{1}=\frac{K_{0}}{B_{1}}, \quad b_{2}=\frac{K_{0}}{B_{2} C_{1}}, c= \\
& \frac{C_{1} B_{2}}{K_{0}}, \quad d_{1}=\frac{D_{1}}{R_{0}}, \quad d_{2}=\frac{D_{2}}{R_{0}}, \text { where } a_{1}=\frac{A_{1} K_{0}}{R_{0} B_{1}}> \\
& b_{1} d_{1}=\frac{K_{0}}{B_{1}} \frac{D_{1}}{R_{0}} \text { or } A_{1}>D_{1} \quad \text { and } a_{2}=\frac{A_{2} K_{0} C_{2}}{B_{2} R_{0} C_{1}}> \\
& b_{2} d_{2}=\frac{K_{0}}{B_{2} C_{1}} \frac{D_{2}}{R_{0}} \text { or } A_{2} C_{2}>,
\end{aligned}
$$

and the non-dimensional version of model (2) is

$$
\begin{gathered}
D_{t}^{\alpha} x=x(1-x)-\frac{a_{1} x y}{1+b_{1} x} \\
D_{t}^{\alpha} y=\frac{a_{1} x y}{1+b_{1} x}-\frac{a_{2} A z y}{1+b_{2} y}-d_{1} y \\
D_{t}^{\alpha} z=\frac{a_{2} A z y}{1+b_{2} y}+(1-A) a_{2} c z-d_{2} z
\end{gathered}
$$

and initial condition is $x(0)=x_{0}, y(0)=y_{0}$, $z(0)=z_{0}$.

\section{Stability of Equilibrium Points}

To determine the equilibrium points of differential equation (3), let 


$$
D_{t}^{\alpha} x=D_{t}^{\alpha} y=D_{t}^{\alpha} z=0,
$$

then the equilibrium points are

$E_{1}=(0,0,0), E_{2}=(1,0,0), E_{3}=(\bar{x}, \bar{y}, 0)$, and

$E_{4}=(\hat{x}, \hat{y}, \hat{z})$ where

$\bar{y}=\frac{a_{1}-\left(b_{1}+1\right) d_{1}}{\left(a_{1}-b_{1} d_{1}\right)^{2}}, \bar{x}=\frac{d_{1}}{a_{1}-b_{1} d_{1}}$,

$\hat{x}=\frac{-\left(1-b_{1}\right)+\sqrt{\left(1-b_{1}\right)^{2}-4 b_{1}\left(\frac{\left(a_{1}+b_{2}\right)\left(d_{2}-a_{2} c(1-A)\right)-a_{2} A}{a_{2} A-\left(d_{2}-a_{2} c(1-A)\right) b_{2}}\right)}}{2 b_{1}}$,

$\hat{y}=\frac{-a_{2} c(1-A)+d_{2}}{a_{2} A-\left(-a_{2} c(1-A)+d_{2}\right) b_{2}}$,

$\hat{z}=\left(\frac{a_{1} \hat{x}}{1+b_{1} \hat{x}}-d_{1}\right)\left(\frac{1}{a_{2} A-\left(a_{2} c(1-A)+d_{2}\right) b_{2}}\right)$.

The Jacobian matrix of system (3) at the equilibrium point $\left(x^{*}, y^{*}, z^{*}\right)$ is given by

$$
J\left(x^{*}, y^{*}, z^{*}\right)=\left(\begin{array}{ccc}
a_{11} & a_{12} & 0 \\
a_{21} & a_{22} & a_{23} \\
0 & a_{32} & a_{33}
\end{array}\right),
$$

where $a_{11}=1-2 x^{*}-\frac{a_{1} y^{*}}{\left(1+b_{1} x^{*}\right)^{2}}, \quad a_{12}=$

$-\frac{a_{1} x^{*}}{1+b_{1} x^{*}}, a_{21}=\frac{a_{1} y^{*}}{\left(1+b_{1} x^{*}\right)^{2}}, a_{22}=\frac{a_{1} x^{*}}{1+b_{1} x^{*}}-$

$\frac{a_{2} A z^{*}}{\left(1+b_{2} y^{*}\right)^{2}}-d_{1}, a_{23}=\frac{a_{2} A y^{*}}{1+b_{2} x^{*}}, a_{32}=\frac{a_{2} A z^{*}}{\left(1+b_{2} y^{*}\right)^{2}}$,

and $a_{33}=\frac{a_{2} A y^{*}}{1+b_{2} y^{*}}+c a_{2}(1-A)-d_{2}$.

Theorem 1. The equilibrium $E_{1}$ of system (3) is always a saddle point.

Proof. The Jacobian matrix at $E_{1}$ is given by

$$
J\left(E_{1}\right)=\left(\begin{array}{ccc}
1 & 0 & 0 \\
0 & -d_{1} & 0 \\
0 & 0 & c a_{2}(1-A)-d_{2}
\end{array}\right) .
$$

Eigenvalues of matrix $J\left(E_{1}\right)$ are obtained by solving the characteristic equation

$$
\begin{aligned}
& P(\lambda)=\operatorname{det}\left(J\left(E_{1}\right)-I \lambda\right) \\
& \quad=(1-\lambda)\left(d_{1}-\lambda\right)\left(c a_{2}(1-A)-d_{2}-\lambda\right)=0 .
\end{aligned}
$$

The eigenvalues corresponding to the equilibrium $E_{1}$ are $\lambda_{1}=1>0, \lambda_{2}=-d_{1}, \quad$ and $\lambda_{3}=$ $c a_{2}(1-A)-d_{2}$. Thus $\left|\arg \left(\lambda_{1}\right)\right|=0<\frac{\alpha \pi}{2}$, $\left|\arg \left(\lambda_{2}\right)\right|=\pi>\frac{\alpha \pi}{2}$, if $d_{2}>c a_{2}(1-A)$ then $\left|\arg \left(\lambda_{3}\right)\right|=\pi>\frac{\alpha \pi}{2}$. Since $\left|\arg \left(\lambda_{1}\right)\right|=0<\frac{\alpha \pi}{2}$,

it follows from convergence of Mittag-Leffler function [9] that the equilibrium $E_{1}$ is always a saddle point.
Theorem 2. The equilibrium $E_{2}$ of system (3) is locally asymptotically stable if $\frac{a_{1}}{1+b_{1}}<d_{1}$ and $c a_{2}(1-A)<d_{2}$.

Proof. The Jacobian matrix of $E_{2}$ is given by

$J(1,0,0)=\left(\begin{array}{ccc}-1 & -\frac{a_{1}}{1+b_{1}} & 0 \\ 0 & \frac{a_{1}}{1+b_{1}}-d_{1} & 0 \\ 0 & 0 & c a_{2}(1-A)-d_{2}\end{array}\right)$.

Eigenvalues of matrix $J\left(E_{2}\right)$ are obtained by solving the characteristic equation

$$
\begin{aligned}
P(\lambda)=\operatorname{det} & \left(J\left(E_{2}\right)-I \lambda\right)=0 \\
= & (-1-\lambda)\left(\frac{a_{1}}{1+b_{1}}-d_{1}-\lambda\right) \\
& \left(c a_{2}(1-A)-d_{2}-\lambda\right)=0 .
\end{aligned}
$$

The eigenvalues corresponding to the equilibrium $E_{2}$ are $\lambda_{1}=-1<0, \lambda_{2}=\frac{a_{1}}{1+b_{1}}-d_{1}$, and $\lambda_{3}=$ $c a_{2}(1-A)-d_{2}$. Thus $\left|\arg \left(\lambda_{1}\right)\right|=\pi>\frac{\alpha \pi}{2}$, if $\frac{a_{1}}{1+b_{1}}<d_{1}$ then $\left|\arg \left(\lambda_{2}\right)\right|=\pi>\frac{\alpha \pi}{2}$, if $d_{2}>$ $c a_{2}(1-A)$ then $\left|\arg \left(\lambda_{3}\right)\right|=\pi>\frac{\alpha \pi}{2}$.

It follows from convergence of Mittag-Leffler function [9] that the equilibrium $E_{2}$ of system (3) is locally asymptotically stable.

Furthermore, the equilibrium points $E_{3}$ and $E_{4}$ are discussed as follows. The Jacobian matrix at $E_{3}$ is given by

$$
J(\bar{x}, \bar{y}, 0)=\left(\begin{array}{ccc}
b_{11} & b_{12} & 0 \\
b_{21} & b_{22} & b_{23} \\
0 & 0 & b_{33}
\end{array}\right) .
$$

where $\quad b_{11}=1-2 \bar{x}-\frac{a_{1} \bar{y}}{\left(1+b_{1} \bar{x}\right)^{2}}, \quad b_{12}=-\frac{a_{1} \bar{x}}{1+b_{1} \bar{x}}$, $b_{21}=\frac{a_{1} \bar{y}}{\left(1+b_{1} \bar{x}\right)^{2}}, b_{22}=\frac{a_{1} \bar{x}}{1+b_{1} \bar{x}}-d_{1}, b_{23}=\frac{a_{2} A \bar{y}}{1+b_{2} \bar{y}^{\prime}}$ and $b_{33}=\frac{a_{2} A \bar{y}}{1+b_{2} \bar{y}}+c a_{2}(1-A)-d_{2}$. Eigenvalues of matrix $J\left(E_{3}\right)$ are $\lambda_{1}=b_{33}=\frac{a_{2} A \bar{y}}{1+b_{2} \bar{y}}+$ $c a_{2}(1-A)-d_{2}$ and the other $\lambda_{2}, \lambda_{3}$ are got by solving the characteristic equation

$$
P(\lambda)=\lambda^{2}-\omega_{1} \lambda+\omega_{2}=0
$$

where $\quad \omega_{1}=1-2 \bar{x}-d_{1}+\frac{a_{1} \bar{x}}{1+b_{1} \bar{x}}-\frac{a_{1} \bar{y}}{\left(1+b_{1} \bar{x}\right)^{2}}$,

$\omega_{2}=\left(1-2 \bar{x}-\frac{a_{1} \bar{y}}{\left(1+b_{1} \bar{x}\right)^{2}}\right)\left(\frac{a_{1} \bar{x}}{1+b_{1} \bar{x}}-d_{1}\right)+$ $\frac{a_{1}^{2} \bar{x} \bar{y}}{\left(1+b_{1} \bar{x}\right)^{3}}$. 
The eigen values corresponding to the equation $P(\lambda)$ are $\lambda_{2}=\frac{1}{2}\left(\omega_{1}+\sqrt{\psi}\right), \lambda_{3}=\frac{1}{2}\left(\omega_{1}-\sqrt{\psi}\right)$, where $\psi=\left(-\omega_{1}\right)^{2}-4 \omega_{2}$. Thus, $E_{3}$ is locally asymptotically stable if it satisfies $\left|\arg \left(\lambda_{1}\right)\right|=$ $\pi>\frac{\alpha \pi}{2}$ by $\frac{a_{2} A \bar{y}}{1+b_{2} \bar{y}}+c a_{2}(1-A)<d_{2}$, and $\lambda_{2}, \lambda_{3}$ are following one of the conditions

1. if $\psi=0$ and $\omega_{1}<0$ then $\lambda_{2}, \lambda_{3}<0$ such that $\left|\arg \left(\lambda_{2,3}\right)\right|=\pi>\frac{\alpha \pi}{2}$,

2. if $\psi>0, \omega_{1}<0, \omega_{2}>0$ and $\sqrt{\psi}<\left|\omega_{1}\right|$ then $\lambda_{2}, \lambda_{3}<0$ such that $\left|\arg \left(\lambda_{2,3}\right)\right|=\pi>$ $\frac{\alpha \pi}{2}$,

3. if $\psi<0$ then $\left|\arg \left(\lambda_{2,3}\right)\right|>\frac{\alpha \pi}{2}$.

To analyze the stability of equilibrium point $E_{4}$, first the Jacobian matrix at $E_{4}$ is evaluated by

$$
J(\hat{x}, \hat{y}, \hat{z})=\left(\begin{array}{ccc}
a_{11} & a_{12} & 0 \\
a_{21} & a_{22} & a_{23} \\
0 & a_{32} & a_{33}
\end{array}\right),
$$

where $\quad a_{11}=1-2 \hat{x}-\frac{a_{1} \hat{y}}{\left(1+b_{1} \hat{x}\right)^{2}}, a_{12}=-\frac{a_{1} \hat{x}}{1+b_{1} \hat{x}^{\prime}}$ $a_{21}=\frac{a_{1} \hat{y}}{\left(1+b_{1} \hat{x}\right)^{2}}, a_{22}=\frac{a_{1} \hat{x}}{1+b_{1} \hat{x}}-\frac{a_{2} A \hat{z}}{\left(1+b_{2} \hat{y}\right)^{2}}-d_{1}$, $a_{23}=-\frac{a_{2} A \hat{y}}{1+b_{2} \hat{y}}, a_{32}=\frac{a_{2} A \hat{z}}{\left(1+b_{2} \hat{y}\right)^{2}}, \quad$ and $\quad a_{33}=$ $\frac{a_{2} A \hat{y}}{1+b_{2} \hat{y}}+c a_{2}(1-A)-d_{2}$. Eigenvalues of matrix $J\left(E_{4}\right)$ are got by solving the characteristic equation

$$
\begin{aligned}
& \begin{array}{l}
P(\lambda)=\operatorname{det}\left(J\left(E_{4}\right)-I \lambda\right) \\
\quad=\lambda^{3}+K_{1} \lambda^{2}+K_{2} \lambda+K_{3}=0
\end{array} \\
& \begin{aligned}
\text { where } \\
K_{1}=-\left(a_{11}+a_{22}+a_{33}\right),
\end{aligned} \\
& \begin{array}{r}
K_{2}=a_{22} a_{33}+a_{11} a_{33}+a_{11} a_{22}-a_{32} a_{23} \\
\quad-a_{21} a_{12},
\end{array} \\
& \begin{array}{c}
K_{3}=a_{32} a_{23} a_{11}+a_{12} a_{23} a_{32}+a_{11} a_{22} a_{33} .
\end{array}
\end{aligned}
$$

Let $D(P)$ is the discriminant of a polynomial $P(\lambda)$, it can be written

$$
\begin{gathered}
D(P)=-\left|\begin{array}{ccccc}
1 & K_{1} & K_{2} & K_{3} & 0 \\
0 & 1 & K_{1} & K_{2} & K_{3} \\
3 & 2 K_{1} & K_{2} & 0 & 0 \\
0 & 3 & 2 K_{1} & K_{2} & 0 \\
0 & 0 & 3 & 2 K_{1} & K_{2}
\end{array}\right|, \\
D(P)=18 K_{1} K_{2} K_{3}+\left(K_{1} K_{2}\right)^{2}-4 K_{3} K_{1}{ }^{2}-4 K_{2}{ }^{3} \\
-27 K_{3}{ }^{2} .
\end{gathered}
$$

\section{Proposition}

Let the equilibrium $E_{4}$ in $\mathbb{R}_{+}^{3}$. Then the equilibrium $E_{4}$ of system (3) is asymptotically stable if one of the following conditions [11] are satisfied

1. $D(P)>0, K_{1}>0, K_{3}>0$,and $K_{1} K_{2}>K_{3}$.

2. $D(P)<0, K_{1} \geq 0, K_{2} \geq 0, K_{3}>0$, and $\alpha<\frac{2}{3}$.
3. $D(P)<0, K_{1}>0, K_{2}>0, K_{1} K_{2}=K_{3}$, and for all $\alpha \in(0,1)$.

\section{Numerical Method and Simulations}

Numerical method which is introduced by Grunwald and Letnikov [8] is used to solve nonlinear fractional differential equation [3]. As described in $[8,12]$, by using the GrunwaldLetnikov approximation method, it is obtained the following nonstandard explicit scheme for system [3].

The parameters chosen in the first

$$
x_{n+1}=h^{\alpha} f\left(x_{n}, y_{n}, z_{n}\right)
$$

$$
\begin{aligned}
& -\sum_{j=0}^{m+1}(-1)^{j}\left(\begin{array}{l}
\alpha \\
j
\end{array}\right) x_{m+1-j} \\
& -\frac{((m+1) h)^{-\alpha}}{\Gamma(1-\alpha)} x_{0} \\
& =h^{\alpha}\left(x_{n}\left(1-x_{n}\right)-\frac{a_{1} x_{n} y_{n}}{1+b_{1} x_{n}}\right) \\
& -\sum_{j=0}^{m+1}(-1)^{j}\left(\begin{array}{l}
\alpha \\
j
\end{array}\right) x_{m+1-j} \\
& -\frac{((m+1) h)^{-\alpha}}{\Gamma(1-\alpha)} x_{0} \\
& =h^{\alpha} g\left(x_{n}, y_{n}, z_{n}\right)-\sum_{j=0}^{m+1}(-1)^{j}\left(\begin{array}{l}
\alpha \\
j
\end{array}\right) y_{m+1-j} \\
& -\frac{((m+1) h)^{-\alpha}}{\Gamma(1-\alpha)} y_{0} \\
& =h^{\alpha}\left(\frac{a_{1} x_{n} y_{n}}{1+b_{1} x_{n}}-\frac{a_{2} A z_{n} y_{n}}{1+b_{2} y_{n}}-d_{1} y_{n}\right) \\
& -\sum_{j=0}^{m+1}(-1)^{j}\left(\begin{array}{l}
\alpha \\
j
\end{array}\right) y_{m+1-j} \\
& -\frac{((m+1) h)^{-\alpha}}{\Gamma(1-\alpha)} y_{0} \\
& z_{n+1}=h^{\alpha} h\left(x_{n}, y_{n}, z_{n}\right)-\sum_{j=0}^{m+1}(-1)^{j}\left(\begin{array}{l}
\alpha \\
j
\end{array}\right) z_{m+1-j} \\
& -\frac{((m+1) h)^{-\alpha}}{\Gamma(1-\alpha)} z_{0} \\
& =h^{\alpha}\left(\frac{a_{2} A z_{n} y_{n}}{1+b_{2} y_{n}}+a_{2} z_{n} c(1-A)-d_{2} z_{n}\right) \\
& -\sum_{j=0}^{m+1}(-1)^{j}\left(\begin{array}{l}
\alpha \\
j
\end{array}\right) z_{m+1-j} \\
& -\frac{((m+1) h)^{-\alpha}}{\Gamma(1-\alpha)} z_{0}
\end{aligned}
$$

simulation are $a_{1}=2, a_{2}=0.1, b_{1}=2.5, b_{2}=$ $2, d_{1}=0.6, \quad d_{2}=0.02, c=0.45, A=0.8$, and $h=0.1$. The aim of this simulation is to show that $E_{2}$ is stable. Figure 1 indicates the different values of $\alpha$ can decide the speed of convergence of solutions. The graph moves from the initial 
condition $(0.5,1.0,0.75))$, then the solution convergent to equilibrium point $\mathrm{E}_{2}(1,0,0)$. If the value of $\alpha$ is approaching to one then the convergence of the rate of change of the three populations is faster and vice versa.
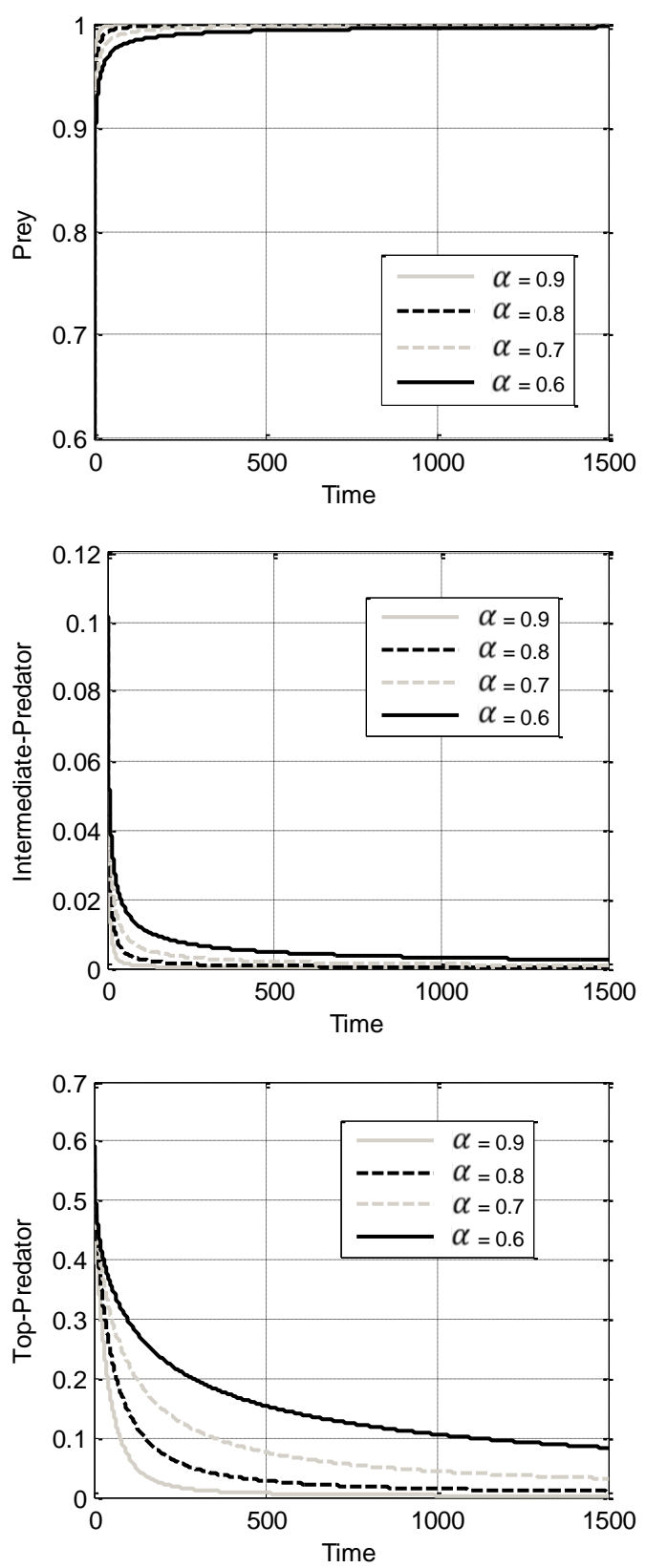

Figure 1 Stability of the equilibrium $E_{1}$ for $\alpha=0.6, \alpha=$ $0.7, \alpha=0.8$ and $\alpha=0.9$

Parameters used in Figure 2 are $a_{1}=5, a_{2}=$ $0.9, b_{1}=3, b_{2}=2.5, d_{1}=0.6, d_{2}=0.3, c=$ $0.45, A=0.6$ and $h=0.1$. According Matignon's condition [13], stability of the equilibrium $E_{3}$ is stabilized by $\alpha^{*}=0.94$. The initial condition of Figure 2 is $(1,0.8,0.5)$ and the solution is stable at point $(0.461,0.414,0)$ for $\alpha=0.9$, and it is unstable for $\alpha=0.95$. From this simulation, it can be concluded that the stability of the equilibrium point of the fractional-order model depends on the parameter of $\alpha$ if $\alpha^{*}>\alpha$ then the equilibrium $E_{3}$ is stable. Conversely if $\alpha^{*}<\alpha$ then the equilibrium $E_{3}$ is unstable.
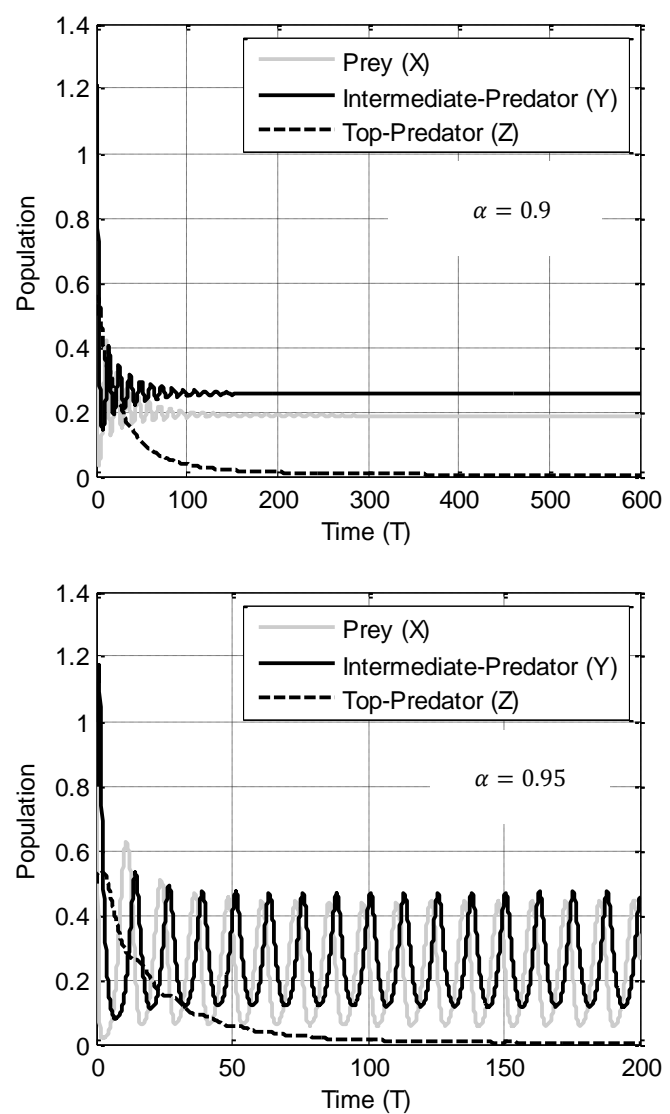

Figure 2. Stability of the equilibrium $E_{3}$ for $\alpha=0.9$ and $\alpha=0.95$

In Figure 3, some parameters are set as $a_{1}=$ $3, a_{2}=0.1, b_{1}=2.5, b_{2}=2, d_{1}=0.6, \quad d_{2}=$ $0.02, c=0.45$, and $h=0.2$. With initial conditions $(1,0.5,1.5)$ and different value of $A=$ $1, A=0.9$, and $A=0.8$, then the solution convergent to $(0.6,0.333,2),(0.71,0.26,2.89)$ and $(0.812,0.189,3.518)$ respectively. These simulations explain that, if there is no alternative food $(A=1)$, the number of top-predator population decreases compared with the presence of alternative food $(0<A<1)$. When the value of $A=1$ indicates that top-predator population doesn't perform activities to search for additional food and the food source of the top-predator population depends only on the intermediate-predator population. However, in this case the three populations still survive in a ecosystem. On the other hand, if top-predator eat only alternative food $(A=0)$ then the top- 
predator can grow without bound (simulation not shown in this paper).
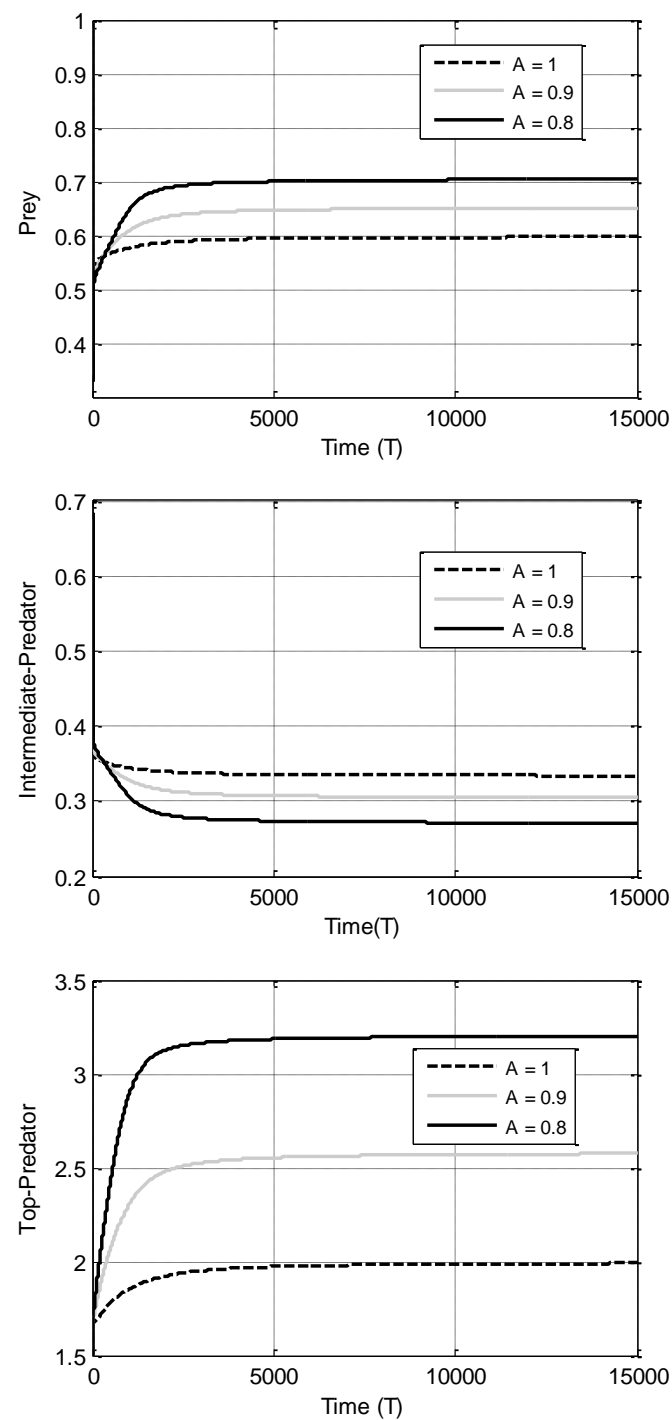

Figure 3. Stability of the equilibrium $E_{4}$ for $\alpha=0.65$ and $A=0.8, A=0.9, A=1$

\section{CONCLUSION}

In this work, the Hastings-Powell food chain model with alternative foods has been modified into a system of fractional-order. The local stability of all the equilibrium points of the fractionalorder system is investigated. Numerical simulation results agree with the analytical result. It is also found that the fractional parameter $\alpha$ has effects on the stability of solution behavior. Furthermore, our analysis predicts that providing a suitable amount of alternative food has a positive impact for top-predator population.

\section{REFERENCES}

[1] Javidi, M., N. Nyamoradi. 2013. Dynamic analysis of a fractional-order prey-predator interaction with harvesting. J. Appl. Math. Model. 37. 8946-8956.

[2] Sahoo, B., S. Poria. 2014. Effects of supplying alternative food in a predator-prey model with harvesting. Appl. Math. Comput. 234. 150-166.

[3] Ilmiyah, N.N., Trisilowati, A.R. Alghofari. 2014. Dynamical analysis of a harvested predator-prey model with ratio-dependent response function and prey refuge. Appl. Math. Sci. 8.5027-5037.

[4] Trisdiani, P.I., Trisilowati, A. Suryanto. 2014. Dynamics of harvested predator-prey system with disease in predator and prey in refuge. Int. J. Ecol. Econ. Stat. 33. 47-57.

[5] Perc., M, A. Szolnoki, G. Szabó. 2007. Cyclical interactions with alliance-specific heterogeneous invasion rates. Phys. Rev. E. 75. 052102.

[6] Perc., M, A. Szolnoki. 2007. Noise-guided evolution within cyclical interactions. New J. Phys. 9. 267.13.

[7] Matouk, A.E., A.A. Elsadany, E. Ahmed, H.N. Agiza. 2015. Dynamical behavior of fractional-order Hastings-Powell food chain model and its discretization. J. Commun Nonlinear Sci. Numer. Simulat. 27. 153-167.

[8] Scherer, R., S.L. Kalla, Y. Tang, J. Huang. 2011. The Grünwald-Letnikov method for fractional differential equations. Comput. Math. Appl. 62. 902-917.

[9] Petras, I. 2011. Fractional-Order Nonlinear systems. Higher Education Press, Beijing and Springer-Verlag Berlin Heidelberg. Beijing.

[10] Hastings, A., T. Powell. 1991. Chaos in threespecies food chain. J. Ecol. 72. 896-903.

[11] Ahmed, E., A.M.A. El-Sayed, H.A.A. El-Saka. 2006. On some Routh-Hurwitz conditions for Fractional-order differential equations and their applications in Lorenz, Rossler, Chua and Chen Systems. Phys. Lett. A. 358. 1-4.

[12] Arenas, A.J., G.G. Parra, B.M ChenCharpentier. 2016. Construction of nonstandard finite difference schemes for the SI and SIR epidemic models of fractional-order. Math. Comput. Simulat. 121. 48-63.

[13] Matignon D. 1996. Stability results for fractional differential equations with applications to control Processing. Proceedings of Computational Engineering in Systems and Application Multi-Conference, Vol. 2: IMACS, IEEE-SMC. Lille, France. 963968. 